\title{
Expression of 16 Nitrogenase Proteins within the Plant Mitochondrial Matrix
}

\author{
Robert S. Allen ${ }^{1}$, Kimberley Tilbrook ${ }^{1}$, Andrew C. Warden ${ }^{2}$, Peter C. Campbell ${ }^{2}$, \\ Vivien Rolland ${ }^{1}$, Surinder P. Singh ${ }^{1}$ and Craig C. Wood ${ }^{1 *}$ \\ ${ }^{1}$ CSIRO Agriculture and Food, Canberra, ACT, Australia, ${ }^{2}$ CSIRO Land and Water, Canberra, ACT, Australia
}

\section{OPEN ACCESS}

Edited by:

Soren K. Rasmussen University of Copenhagen, Denmark

Reviewed by:

Philip Simon Poole,

University of Oxford, UK Ian Max Moller

Aarhus University, Denmark

*Correspondence:

Craig C. Wood

craig.wood@csiro.au

Specialty section:

This article was submitted to

Plant Biotechnology,

a section of the journal

Frontiers in Plant Science

Received: 17 October 2016

Accepted: 16 February 2017

Published: 03 March 2017

Citation:

Allen RS, Tilbrook K, Warden AC, Campbell PC, Rolland V, Singh SP and Wood CC (2017) Expression of 16 Nitrogenase Proteins within the

Plant Mitochondrial Matrix.

Front. Plant Sci. 8:287.

do: 10.3389/fp/s.2017.00287
The industrial production and use of nitrogenous fertilizer involves significant environmental and economic costs. Strategies to reduce fertilizer dependency are required to address the world's increasing demand for sustainable food, fibers, and biofuels. Biological nitrogen fixation, a process unique to diazatrophic bacteria, is catalyzed by the nitrogenase complex, and reconstituting this function in plant cells is an ambitious biotechnological strategy to reduce fertilizer use. Here we establish that the full array of biosynthetic and catalytic nitrogenase (Nif) proteins from the diazotroph Klebsiella pneumoniae can be individually expressed as mitochondrial targeting peptide (MTP)-Nif fusions in Nicotiana benthamiana. We show that these are correctly targeted to the plant mitochondrial matrix, a subcellular location with biochemical and genetic characteristics potentially supportive of nitrogenase function. Although Nif proteins $B, D, E, F, H, J, K, M$, $\mathrm{N}, \mathrm{Q}, \mathrm{S}, \mathrm{U}, \mathrm{V}, \mathrm{X}, \mathrm{Y}$, and Z were all detectable by Western blot analysis, the NifD catalytic component was the least abundant. To address this problem, a translational fusion between NifD and NifK was designed based on the crystal structure of the nitrogenase MoFe protein heterodimer. This fusion protein enabled equimolar NifD:NifK stoichiometry and improved NifD expression levels in plants. Finally, four MTP-Nif fusion proteins (B, $\mathrm{S}, \mathrm{H}, \mathrm{Y}$ ) were successfully co-expressed, demonstrating that multiple components of nitrogenase can be targeted to plant mitochondria. These results establish the feasibility of reconstituting the complete componentry for nitrogenase in plant cells, within an intracellular environment that could support the conversion of nitrogen gas into ammonia.

Keywords: nitrogenase, synthetic biology, nitrogen fixation, metabolic engineering, mitochondrial targeting

\section{INTRODUCTION}

Diazotrophic bacteria produce ammonia from $\mathrm{N}_{2}$ gas via biological nitrogen fixation (BNF), catalyzed by nitrogenase. Yet the demands of modern agriculture far outstrip this source of fixed nitrogen, and industrially-produced nitrogenous fertilizer (INF, Smil, 2002) is used extensively in agriculture. However, both INF production and application are causes of pollution (Good and Beatty, 2011) and considered overall unsustainable (Rockstrom et al., 2009). Approximately half the fertilizer applied worldwide is not taken up by crops (Cui et al., 2013; Kronzucker and Coskun, 2015), leading to fertilizer runoff, promotion of weeds and eutrophication of waterways (Good and Beatty, 2011). Resultant algal blooms reduce oxygen levels, causing environmental damage locally 
and offshore throughout coral reefs (Sutton et al., 2008; De'ath et al., 2012; Glibert et al., 2014). Furthermore, although overfertilization is a problem in many developed countries, in certain regions fertilizer availability limits crop yields (Mueller et al., 2012).

Strategies to reduce the global dependence on nitrogen fertilizers need to be explored, and biotechnological approaches have been suggested. To this end, the notion of engineering plants capable of BNF has long attracted considerable interest (Merrick and Dixon, 1984), and has been the focus of recent reviews (Oldroyd and Dixon, 2014; de Bruijn, 2015). Potential approaches include (i) extending the symbiotic relationship of diazotrophs from legumes to cereals (Santi et al., 2013), (ii) re-engineering endosymbiotic microorganisms to be capable of nitrogen fixation (Geddes et al., 2015), and (iii) genetic engineering of nitrogenase into plant cells (Curatti and Rubio, 2014). Whilst all of these approaches are ambitious we outline here our first steps toward the direct engineering of nitrogenase into the mitochondrial matrix of plants.

Nitrogenase, the unique enzyme complex capable of BNF in diazotrophic bacteria, requires a multigene assembly pathway for its biosynthesis and function, reviewed extensively previously (Rubio and Ludden, 2008; Seefeldt et al., 2009; Hu and Ribbe, 2013). There are two subunits of the canonical iron-molybdenum nitrogenase, the catalytic MoFe subunit comprised of NifD and NifK proteins, and the electron donor Fe subunit comprised of NifH. A further series of proteins is involved in electron transport and nitrogenase assembly (maturation, scaffolding, cofactor insertion), including Nif $\mathrm{B}, \mathrm{M}, \mathrm{S}, \mathrm{U}, \mathrm{E}, \mathrm{N}, \mathrm{X}, \mathrm{V}, \mathrm{J}, \mathrm{Y}$, F, Z, and Q. Specific biochemical conditions are also required for nitrogenase assembly and function. Foremost among these is the necessity for protection from oxygen, as nitrogenase is extremely oxygen sensitive (Robson and Postgate, 1980). Furthermore, large amounts of ATP, reductant, readily available $\mathrm{Fe}, \mathrm{Mo}$, S-adenosylmethionine, and homocitrate are required for biosynthesis and function of the metalloprotein complex (Rubio and Ludden, 2008; Hu and Ribbe, 2013).

Given these requirements for nitrogenase function, the mitochondrial matrix is considered a suitable location for its reconstitution and activity (Curatti and Rubio, 2014). Significantly, the matrix possess oxygen-consuming enzymes that allow oxygen-sensitive enzymes to function, therefore this environment may be similarly permissive for nitrogenase activity. Secondly as the major site of plant metalloenzyme synthesis, mitochondria contain biosynthetic assembly proteins, which could provide the functionality of equivalent Nif proteins (Lill and Mühlenhoff, 2008; Balk and Pilon, 2011). These concepts have been partially validated by the successful isolation of an ex vivo active Nif Fe subunit from the mitochondrial matrix of aerobically grown yeast (López-Torrejón et al., 2016). This breakthrough result indicates that the matrix may support the assembly and activity of a complete nitrogenase. Nevertheless, an active nitrogenase in any eukaryote will require a much larger array of Nif proteins to be co-expressed, including the catalytic core.

As a first step toward reconstitution of nitrogenase in plant mitochondria, evidence is needed that individual Nif proteins can be correctly targeted to the organelle. For this purpose we made use of the model plant Nicotiana benthamiana, where metabolic engineers have developed an expression platform that allows single and multiple transgenes to be expressed and assayed within a week (Wood et al., 2009). As most matrix-located proteins are nuclear-encoded, we have relied upon recent advances in understanding of subcellular signaling and transport of proteins (Huang et al., 2009; Murcha et al., 2014), using a previously characterized $\mathrm{N}$-terminal peptide targeting signal (Lee et al., 2012). Here we re-engineer 16 Nif proteins from diazotrophic Klebsiella pneumoniae for targeting to the plant matrix and assess their expression and processing in $N$. benthamiana. Our results are discussed in the context of recent advances in nitrogenase engineering in subcellular locations of plants and yeast.

\section{RESULTS}

\section{Validation of a Mitochondrial Targeting Peptide for Directing Nif Proteins into $N$. benthamiana Leaf Mitochondria}

An Arabidopsis thaliana F1-ATPase pFA $\gamma$ subunit mitochondrial targeting peptide (MTP; pFA $\gamma$ ) that has previously been functionally validated in Arabidopsis protoplasts (Lee et al., 2012) was tested for its ability to traffic transgenic proteins to the matrix of intact plant leaf cells using the $N$. benthamiana transient leaf assay system. The entire pFA $\gamma$ (77 AA) as previously described was translationally fused to the $\mathrm{N}$ terminus of GFP (pFA $\gamma:: \mathrm{GFP})$, where the first $42 \mathrm{AA}(\sim 4.6 \mathrm{kDa})$ of pre-sequence is predicted to be removed via the matrix-located peptidase (Figure 1A). As a control to discriminate matrix-processed GFP, several alanine amino acid substitutions were introduced in regions of the MTP required for its mitochondrial recognition and processing by the mitochondrial processing peptidase (MPP; Lee et al., 2012), thus this version (mFA $\gamma::$ GFP) should be unable to be correctly processed and rather produce a full length fusion protein $\sim 4.6 \mathrm{kDa}$ larger than $\mathrm{pFA} \gamma:: \mathrm{GFP}$. Five-week-old $N$. benthamiana leaves were infiltrated with either pFA $\gamma:$ GFP or $\mathrm{mFA} \gamma:: \mathrm{GFP}$. SDS-polyacrylamide gel electrophoresis (PAGE) and Western blots were carried out on crude protein extracted 4 days post infiltration (4 dpi) using a GFP antibody. For pFA $\gamma:: G F P$, a band was observed corresponding to the expected size $(\sim 30 \mathrm{kDa})$ for matrix-processed GFP polypeptide, whereas for mFA $\gamma:: G F P$, a higher MW band was observed $(\sim 35 \mathrm{kDa})$ of the expected size for unprocessed GFP fusion polypeptide (Figure 1B). A fainter band at $\sim 28 \mathrm{kDa}$ was observed for both pFA $\gamma::$ GFP and mFA $\gamma::$ GFP. This was not observed in negative controls and therefore may represent a degradation product, or possibility a product arising from alternative transcription or translation. To determine the subcellular localization of the GFP fusion proteins, protoplasts were isolated $3 \mathrm{dpi}$ from leaf tissues containing either pFA $\gamma:: \mathrm{GFP}, \mathrm{mFA} \gamma:: \mathrm{GFP}$, or no vector, and were examined by confocal microscopy after staining their mitochondria with MitoTracker. In protoplasts expressing $\mathrm{pFA} \gamma:: \mathrm{GFP}$, the GFP signal fully co-localized with the mitochondrial marker (Figures 1G-J, white arrowheads) and was absent in control protoplasts (Figures 1C-F). In contrast, in 
A

$\sim 4.6 \mathrm{kDa}$

MAMAVFRREGRRLLPSIAARPIAAIRSPLSSDQEEGLLGVRS I STQVVRNRMKSVKNIQKITKAMKMVAASKLRAVQ

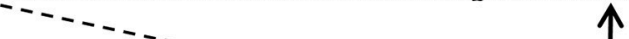

$35 S$ Pr

mFA $\gamma$

MAMAVFRREGR AAAAAAAARPIA AAAAAAAAAAEEGLI AAAAAAABVVRNRMKSVKNIQKITKAMKMVAASKLRAV̈:

B
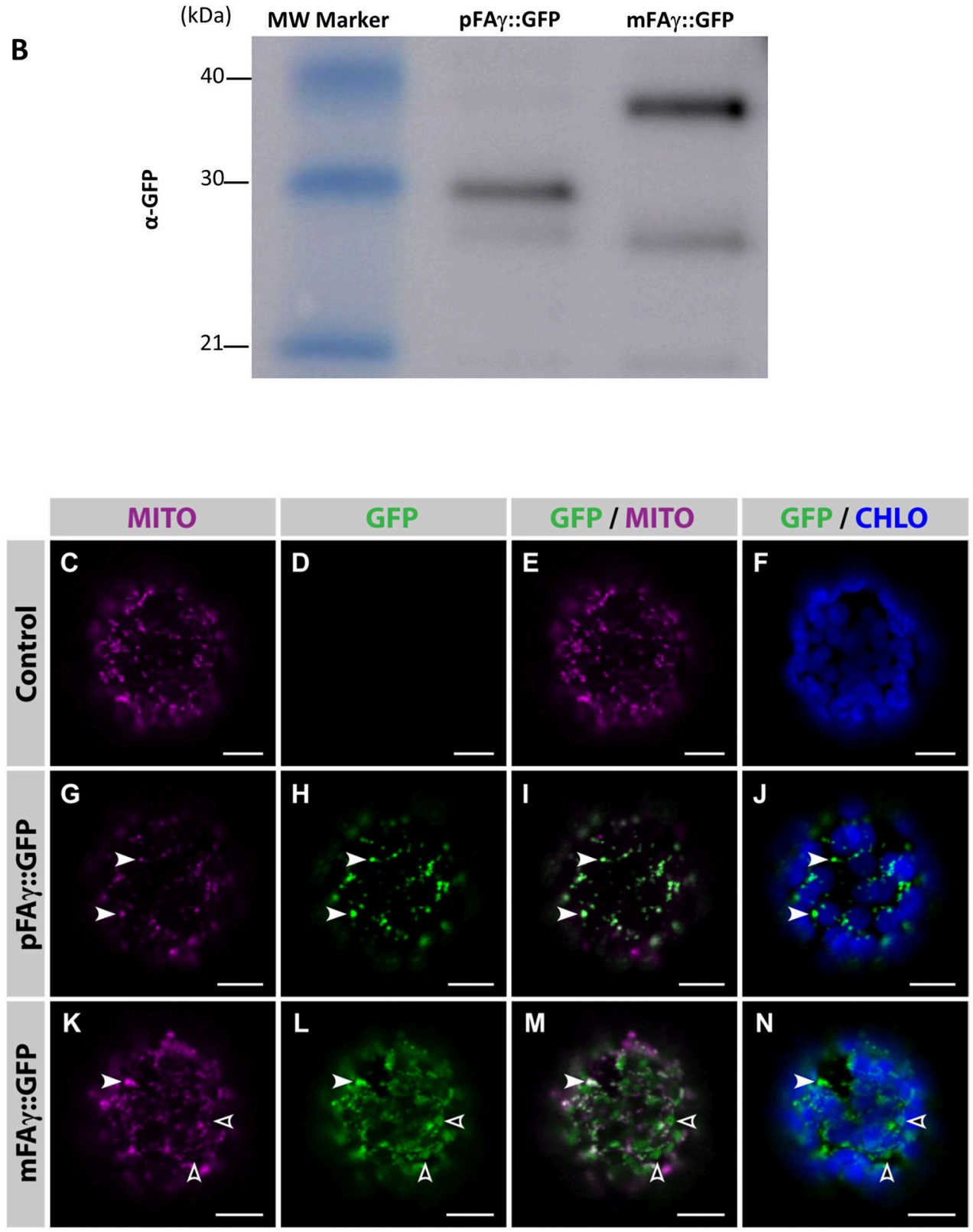

FIGURE 1 | Validation of the MTP for mitochondrial matrix targeting in $\mathbf{N}$. benthamiana leaves. (A) Schematic diagram of the constructs used to transiently express pFA $\gamma:$ :GFP fusion polypeptides in N. benthamiana leaves. The wild-type pFA $\gamma$ mitochondrial targeting peptide sequence (MTP) shown above and the mutated 


\section{FIGURE 1 | Continued}

version (mFA $\gamma$ ) shown below. Red boxes indicate regions of alanine substitutions. The arrow indicates the predicted point of cleavage by the MPP. 35S Pr, CaMV $35 \mathrm{~S}$ promoter; T7Pr, T7 RNA polymerase promoter; MTP, pFA $\gamma$, or mFA $\gamma$ region; GFP, GFP polypeptide; T7Tm, T7 RNA polymerase transcription terminator; NOSTm, 3' transcription terminator/polyadenylation region of the nos gene. (B) Western blot of protein extracts for constructs expressing pFA $\gamma:$ GFP or mFA $\gamma:$ GFP fusion polypeptides in $N$. benthamiana leaves. Molecular weights of the markers in the first lane are indicated. The band in the pFA $\gamma:$ GFP lane is the cleaved fusion polypeptide, whereas the intense band in the mFA $\gamma:$ GFP lane is the uncleaved fusion polypeptide. The GFP antibody also produces a second slightly fainter lower band of $\sim 28 \mathrm{kDa}$. (C-N) Laser scanning confocal microscopy images of protoplasts isolated 3 dpi from $N$. benthamiana leaves that were either non-infiltrated controls (C-F), or infiltrated with pFA $\gamma:$ :GFP (G-J) or mFA $\gamma:$ :GFP (K-N). In protoplasts expressing pFA $\gamma:$ GFP (G-J), the GFP signal fully co-localized with MitoTracker (white arrowheads), while in protoplasts expressing mFA $\gamma:$ GFP (K-N), the GFP signal only partially co-localized with MitoTracker (white arrowheads,) and a large fraction of GFP was mis-targeted to other subcellular areas (empty arrowheads). MITO, MitoTracker fluorescence; GFP, GFP fluorescence; GFP/MITO, overlay of GFP and MitoTracker staining fluorescence; GFP/CHLO overlay of GFP and chloroplast fluorescence. Scale bars: $10 \mu \mathrm{m}$.

protoplasts expressing mFA $\gamma::$ GFP the GFP signal only partially localized to mitochondria (Figures $1 \mathbf{K}-\mathbf{N}$, white arrowheads), and a large fraction of the GFP signal was found to be targeted to other subcellular areas (Figures $\mathbf{1 K}-\mathbf{N}$, open arrowheads). Taken together these analyses indicate that the pFA $\gamma$ MTP was capable of translocating the GFP fusion polypeptide to the matrix in $N$. benthamiana leaf cells and cleavage by the MPP, while the use of mFA $\gamma$ MTP resulted in largely mis-targeted fusion proteins.

We next wanted to test if the pFA $\gamma$ MTP was capable of directing bacterial nitrogenase proteins to the matrix. For this, two Nif proteins were chosen (NifF and NifZ) because their relatively small sizes would enable clear discrimination of the processed size by Western blotting. Protein-coding regions for the $K$. pneumoniae NifF and NifZ polypeptides were codon optimized for eukaryotic expression and fused to either HA or FLAG epitopes as C-terminal fusions (Figures 2A,B and Supplementary Table 1). The pFA $\gamma$ MTP was fused to the $\mathrm{N}$ terminus of these polypeptides as for GFP above. To generate unprocessed versions of these pFA $\gamma:$ : Nif proteins the same constructs were expressed in Escherichia coli by T7 RNA polymerase. Given that the MTP cannot be processed in bacteria which have neither mitochondria nor MPP, the difference in size between plant and bacterially- expressed proteins would enable processing to be validated by SDS-PAGE and Western blot. The Western blot revealed that the size of the polypeptides extracted from the $N$. benthamiana leaves was smaller in each case than the corresponding polypeptide produced in E. coli (Figures 2A,B). For each of pFA $\gamma::$ NifF::HA and pFA $\gamma:$ NifZ::FLAG, the polypeptides extracted from the plant cells corresponded to the sizes predicted for cleavage of the fusion polypeptides in their MTPs, whereas the polypeptides detected in E. coli extracts were of the expected sizes for unprocessed $\mathrm{pFA} \gamma::$ Nif fusion polypeptides. From these data we could conclude that the pFA $\gamma$ MTP was capable of both directing Nif-fused polypeptides into the matrix and cleavage of the MTP by the MPP in plant cells.

Finally, we validated that the predicted MTP processing site was cleaved using mass spectrometer (MS) analysis. For this we chose NifH-HA, due to its importance as a core component of the nitrogenase enzyme complex. pFA $\gamma:: \mathrm{NifH}:: \mathrm{HA}$ was expressed in plants, immunoprecipitated on HA-antibody agarose, further enriched via denaturing SDS electrophoresis (Figure 2C), and subjected to in-gel digestion with trypsin followed by tandem MS analysis of the resultant peptides. This analysis found 5 fully tryptic peptides identical to regions within $\mathrm{NifH}$ and a sixth semi-tryptic peptide consistent with exact cleavage of the MTP between residues 42 and 43 (Figure 2D). The tryptic peptide SISTQVVR that would been obtained from an unprocessed MTP was not observed. Instead, the most $\mathrm{N}$-terminal peptide that was detected was the semi-tryptic ISTQVVR, confirmed by a complete series of $y$-ions in its MS/MS spectrum. These data conclusively demonstrated that the pFA $\gamma:$ NifH::HA polypeptide had been cleaved at the preselected site in the MTP within the Nterminal extension by the matrix processing protease. The data implied that the pFA $\gamma$ MTP contained all of signals necessary for translocation and processing in the matrix.

\section{Expression of Individual Nitrogenase Proteins in the Mitochondrial Matrix of Plants}

Given the success of expression, detection, and processing of GFP, NifF, NifZ, and NifH polypeptides in N. benthamiana mitochondria, we attempted to determine if all remaining Nif proteins required for nitrogenase biosynthesis and function could be expressed in $N$. benthamiana mitochondria. In the model diazatroph $K$. pneumoniae, 16 Nif proteins are involved in nitrogenase biosynthesis or catalysis, while four others are of unknown function or involved in transcriptional regulation (Oldroyd and Dixon, 2014). We therefore chemically synthesized eukaryotic expression codon-optimized versions of all these essential biosynthetic and catalytic nif genes of K. pneumoniae, each fused to the previously validated pFA $\gamma$ MTP (Figure 3A, Supplementary Table 1). Each genetic construct encoded a fusion polypeptide having an N-terminal pFA $\gamma$ MTP, then the Nif sequence, followed by a C-terminal extension comprising either an HA or FLAG epitope for detection by the appropriate antibody. For plant expression, each construct included the $35 \mathrm{~S}$ promoter and nopaline synthase (nos) $3^{\prime}$ transcription terminator regions flanking the protein coding region.

A. tumefaciens cells containing each of the 16 genetic constructs were separately infiltrated into $N$. benthamiana leaves and, 4 days later, protein extracts prepared and analyzed by SDS-PAGE and Western blotting as before. For each of the constructs encoding HA-tagged pFA $\gamma$-Nif polypeptides, bands were detected on the Western blots which were approximately the size as predicted for the matrix-processed polypeptide (Figure 3B). Protein abundance varied among the HA-tagged polypeptides, with the easiest to detect being the $\mathrm{NifB}, \mathrm{NifH}$, 
A

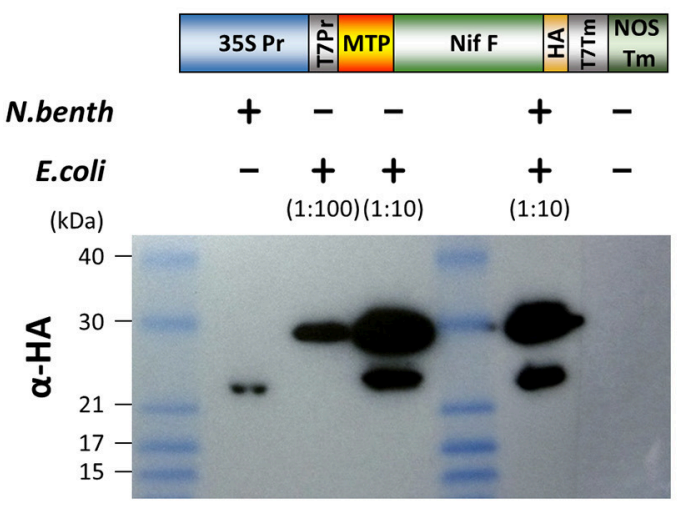

B

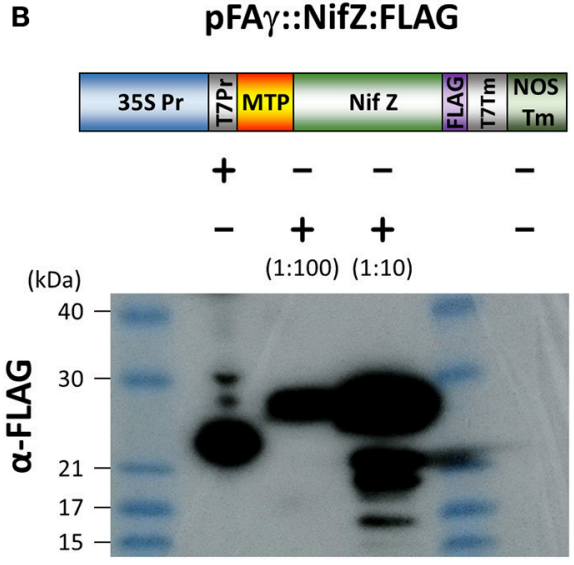

C

pFA $\gamma:$ NifH:HA

pFA $\gamma:: G F P$

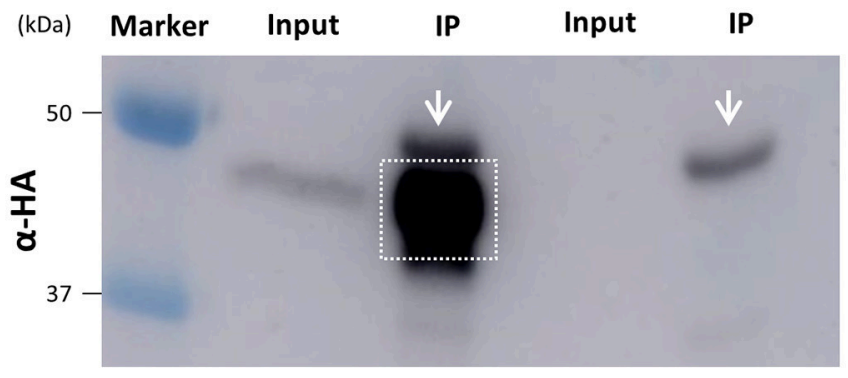

D

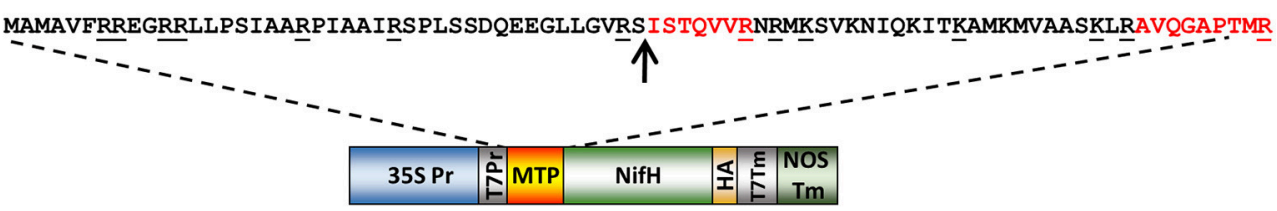

FIGURE 2 | Expression and processing of mitochondrially targeted Nif proteins in $\mathbf{N}$. benthamiana. (A,B) Schematic of the pFA $\gamma:$ NifF::HA and pFA $\gamma:$ NifF::FLAG constructs used for $N$. benthamiana and $E$. coli expression, indicating the T7 promoter driving bacterial expression downstream of the $35 S$ promoter for plant expression. Western blot analysis of pFA $\gamma:$ NifF::HA or pFA $\gamma:$ NifF::FLAG expression in $N$. benthamiana and $E$. coli. The + and - symbols above the lanes indicate the presence or absence, respectively, of $N$. benthamiana or $E$. coli protein extracts applied to the lanes; the extract dilution factors used for the bacterial extracts are indicated in brackets. (C) Image of Western blot for protein concentration of pFA $\gamma:$ NifF::HA by anti-HA immunoprecipitation. Original protein extracts (Input) and IP eluate (IP) are shown. Background signal from the large-chain subunit of the HA antibody is marked with white arrow. The gel area excised for protein microsequencing is indicated by the white box. (D) Schematic of the construct used to transiently express pFA $\gamma:$ NifH::HA in N. benthamiana. Underlined residues in the nucleotide sequence indicate sites of proteolytic cleavage (carboxyl side) by trypsin. The arrow indicates the point of cleavage by the mitochondrial processing peptidase (MPP). The peptides ISTQVVR and AVQGAPTMR were detected by mass spectrometry.

NifK, NifS, and NifY fusion polypeptides. The NifF, NifE, and NifM polypeptides were present at lower levels, whereas detection of the NifQ polypeptide required a longer exposure of the blot to be visible (Supplementary Figure 1). Interestingly, additional, higher-molecular weight bands were detected for the infiltrations with the NifB, NifS, NifH, and NifY constructs that were size-specific to each individual construct. These additional bands were approximately twice the molecular weight of the primary band, suggesting that these polypeptides were dimerising despite the denaturing conditions during gel electrophoresis. It has been reported the $\mathrm{NifB}$, NifS, and $\mathrm{NifH}$ proteins function as homo-dimers in bacteria (Yuvaniyama et al., 2000; Rubio and Ludden, 2008).

FLAG-tagged pFA $\gamma::$ Nif::FLAG fusion polypeptides were observed in the Western blots for each of the constructs including the NifJ, NifN, NifV, NifU, NifX, and NifZ sequences (Figure 3B). The FLAG antibody yielded more background bands from the $N$. benthamiana extracts than the HA antibody. Nevertheless, the results were similar to those for the HAtagged proteins. Considerable variation was seen in signal intensity for the different pFA $\gamma:$ :Nif::FLAG fusion polypeptides. An additional, higher-molecular-weight band was observed 

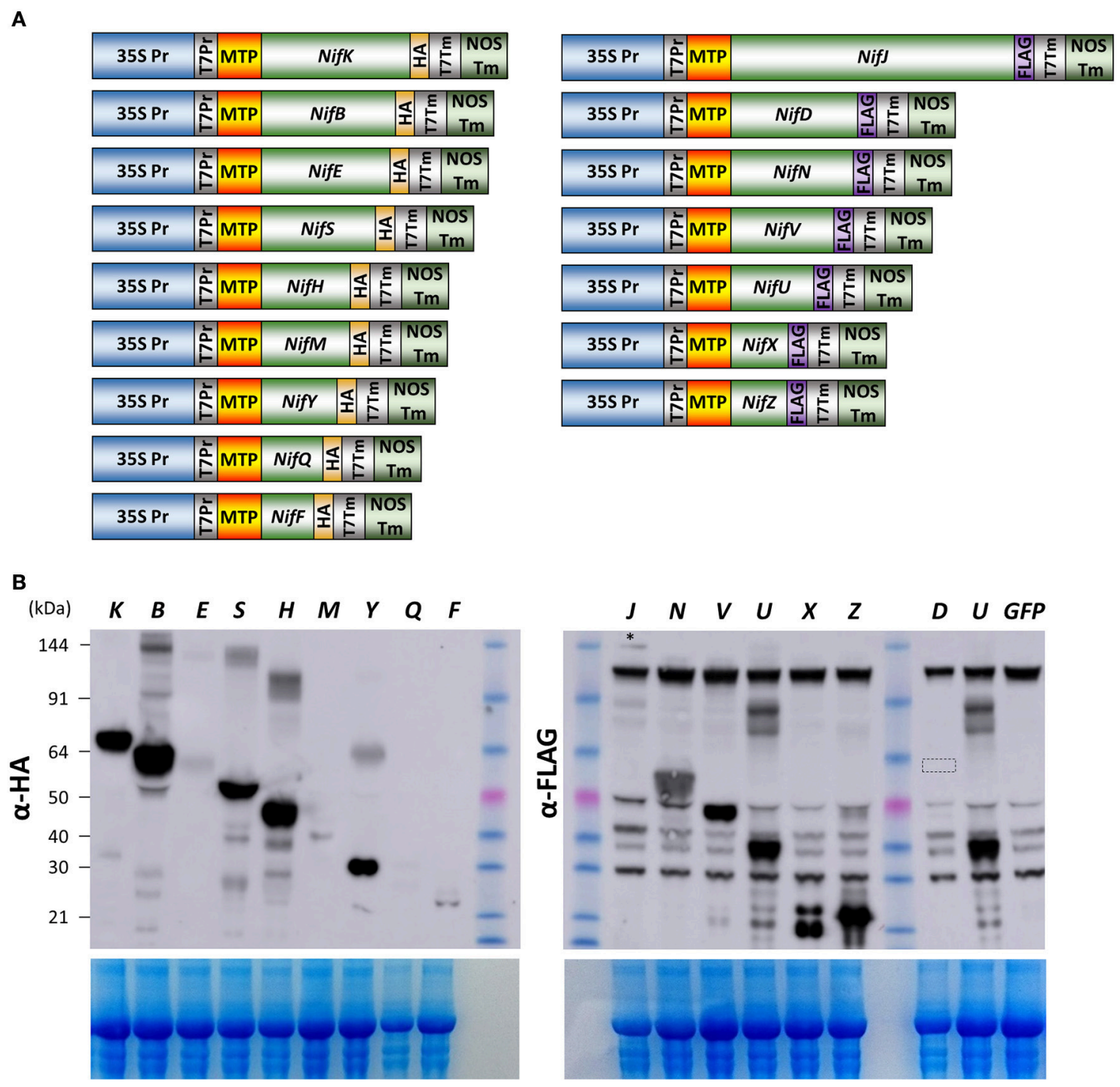

FIGURE 3 | Expression of 15 Nif proteins in the mitochondrial matrix of $\boldsymbol{N}$. benthamiana. (A) Schematics of the constructs used for pFA $\gamma:$ Nif::HA and pFAy::Nif::FLAG expression in N. benthamiana. Only the Nif inserts are relatively proportional to their sequence length. (B) Image of Western blot probed with antibody for HA (upper left panel) or FLAG (upper right panel) after SDS-PAGE of protein extracts from N. benthamiana cells expressing constructs encoding pFA $;::$ Nif::HA or pFAy::Nif::FLAG fusion polypeptides. The letters above the lanes (K, B, E, S etc.) indicate the Nif polypeptide included in the fusion polypeptide encoded by the genetic construct. The faint band near the top of the blot for pFA $\gamma:$ NifJ::FLAG is indicated by an asterisk (*). A small box in the Lane "D" highlights the region of the blot where a signal for pFA $\gamma:$ NiD::FLAG would be expected. The extreme right lane GFP indicates a sample extracted from a pFA $\gamma:$ GFP infiltrated region as a negative control for background bands inherent to the FLAG epitope in these assays. The size of the molecular weight markers (kDa) are indicated to the left, and the same markers were used in both HA and FLAG panels. The expected sizes for processed and unprocessed proteins are shown in Supplementary Table 1. The lower panels show the corresponding gels after Coomassie staining as an indication of protein loading.

that was specific for the NifU construct (Figure 3B). The pFA $\gamma::$ NifX::FLAG construct also yielded an additional, smaller band of greater intensity than expected for the predicted, processed molecular weight. Despite numerous replications of the infiltrations, we were unable to detect any specific bands for the pFA $\gamma:: N i f D:: F L A G$ construct in these plant assays, even though expression of the same genetic construct in E. coli readily yielded a visible band of the expected molecular weight (Figure 4A). The result from the bacterial extracts confirmed that the genetic construct containing pFA $\gamma:: N i f D:: F L A G$ was translationally competent.

\section{Improved MTP-NifD Production in N. benthamiana}

Given the key role of NifD in the catalytic activity of nitrogenase (Eady et al., 1972), we tried to identify the reason for the lack of NifD fusion polypeptide production and tested several 


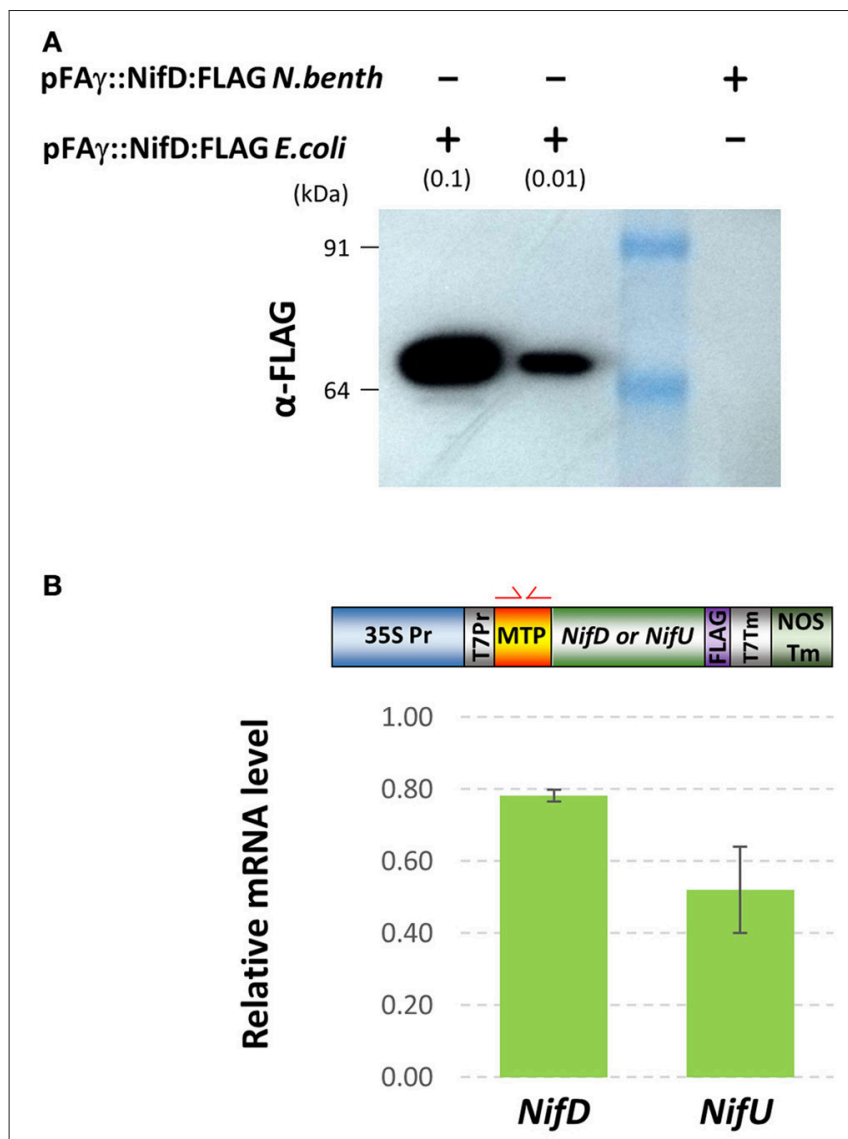

C

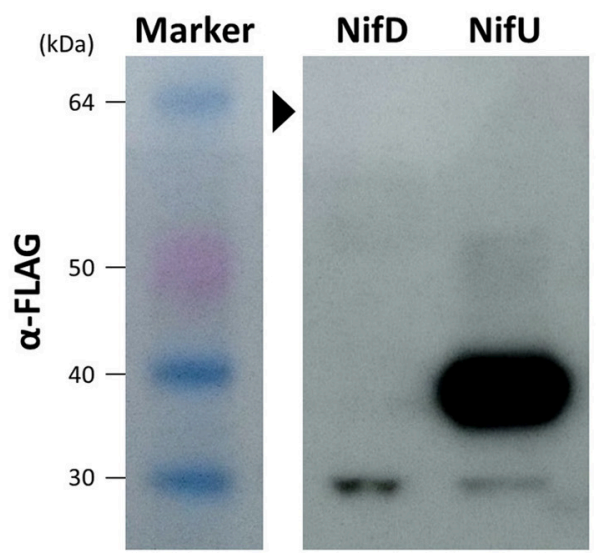

FIGURE 4 | Expression of MTP-NifD-FLAG is undetectable in N.benthamiana despite high relative mRNA expression. (A) Western blot of anti-FLAG for pFA $\gamma:$ NifD::FLAG transformed $E$. coli or N. benthamiana. The blot was probed with the antibody against the FLAG epitope. The molecular weights of the markers in the first lane are indicated. The + and - symbols above the lanes indicate the presence or absence, respectively, of $N$. benthamiana or E. coli protein extracts applied to the lanes; the extract dilution factors used for the bacterial extracts are indicated in brackets. (B) qRT-PCR analysis of $p F A \gamma:$ Nif::FLAG transgene expression. Schematic above shows the primers annealing in the MTP region. Expression values were normalized to GADPH, with measurements being the average of three replicates and error bars representing the standard error of the mean.

(Continued)

\section{FIGURE 4 | Continued}

(C) Image of Western blot using anti-FLAG for protein extracts prepared from $N$. benthamiana leaf samples 4 days after infiltration with either pFA $\gamma::$ NifD::FLAG or pFA $\gamma::$ NifU::FLAG. Black arrowhead indicates the expected position for a pFA $\gamma:$ NifD::FLAG band. Protein marker sizes are indicated on left hand side of image.

approaches to improve its abundance in plant assays. Firstly, we tested whether the lack of MTP-NifD fusion polypeptide production/accumulation could be attributed to low transgene transcription or mRNA instability, by measuring the mRNA expression level. To do this, the level of mRNA in the infiltrated $N$. benthamiana cells from $\mathrm{pFA} \gamma:$ :NifD::FLAG was first measured by qRT-PCR and compared to the level of mRNA transcribed from the construct encoding pFA $\gamma:$ :NifU::FLAG in $N$. benthamiana cells (Figure 4B). This second construct was used as a control since it provided high levels of polypeptide production in the plant cells, as described above (Figure 4C). In order to remove any bias in amplification efficiency, oligonucleotide primers were used which annealed within the pFA $\gamma$ MTP region shared by both nif fusion genes (illustrated in Figure 4B, Supplementary Table 2). The results from the qRT-PCR assay showed that the level of $p F A \gamma::$ nifD::FLAG mRNA was actually slightly higher, although not significantly $(P=0.0683)$, than the level of $p F A \gamma:: n i f U:: F L A G$ mRNA. Secondly, cDNA was synthesized from the plantproduced mRNA transcribed from $p F A \gamma:: n i f D:: F L A G$, cloned and sequenced, and its nucleotide sequence proved to be base perfect. Therefore, the lack of accumulation of the pFA $\gamma::$ NifD::FLAG fusion polypeptide was not due to low mRNA expression or instability. These experiments also showed that the T-DNA containing and encoding pFA $\gamma:: N i f D:: F L A G$ was fully functional and that the $35 \mathrm{~S}$ promoter in that construct was likewise functional. Therefore, the inability to detect the MTPNifD fusion polypeptide in $N$. benthamiana cells was not due to any lesions in expression of the mRNA.

As transcription of the gene encoding pFA $\gamma::$ NifD::FLAG and mRNA accumulation was clearly not limiting MTPNifD production, several modifications were made to the genetic construct in attempts to improve protein accumulation (Figure 5A). Firstly, the possibility was tested that the presence of the FLAG epitope in the C-terminal extension was causing either lack of production or instability of the NifD fusion polypeptide. For this, a construct was designed in which the FLAG epitope was substituted with an HA epitope, designated pFA $\gamma:: N i f D:: H A$. The HA epitope had allowed for accumulation and detection of 9 other MTP-Nif proteins (Figure 3B). Secondly, the codon usage of the NifD::HA open reading frame was modified in an attempt to determine whether a different $m R N A$ sequence might improve translation efficiency. For this, an A. thaliana codon optimization algorithm was utilized (Graf et al., 2009), whereas the previous construct used a H. sapien algorithm (Graf et al., 2009). This construct was designated $\mathrm{pFA} \gamma:: \mathrm{NifD}_{\mathrm{At}}:: \mathrm{HA}$, and encoded an identical polypetide to $\mathrm{pFA} \gamma:$ NifD::HA. Additionally, a genetic construct was made encoding a version of the NifD fusion polypeptide with the mutated $\mathrm{mFA} \gamma \mathrm{N}$-terminal extension rather 
A

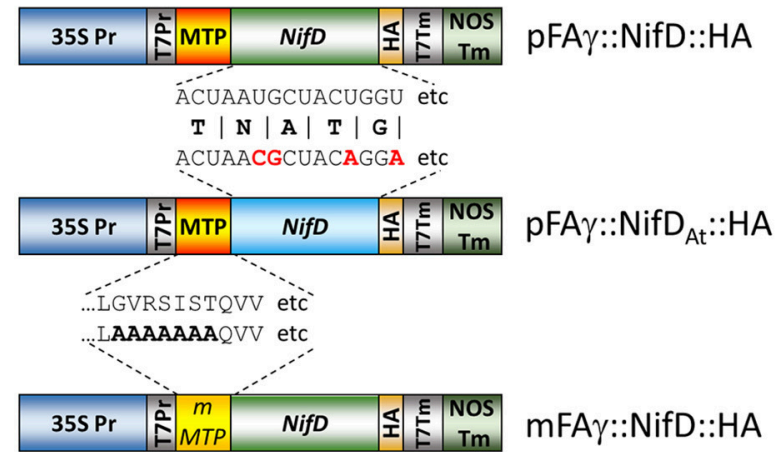
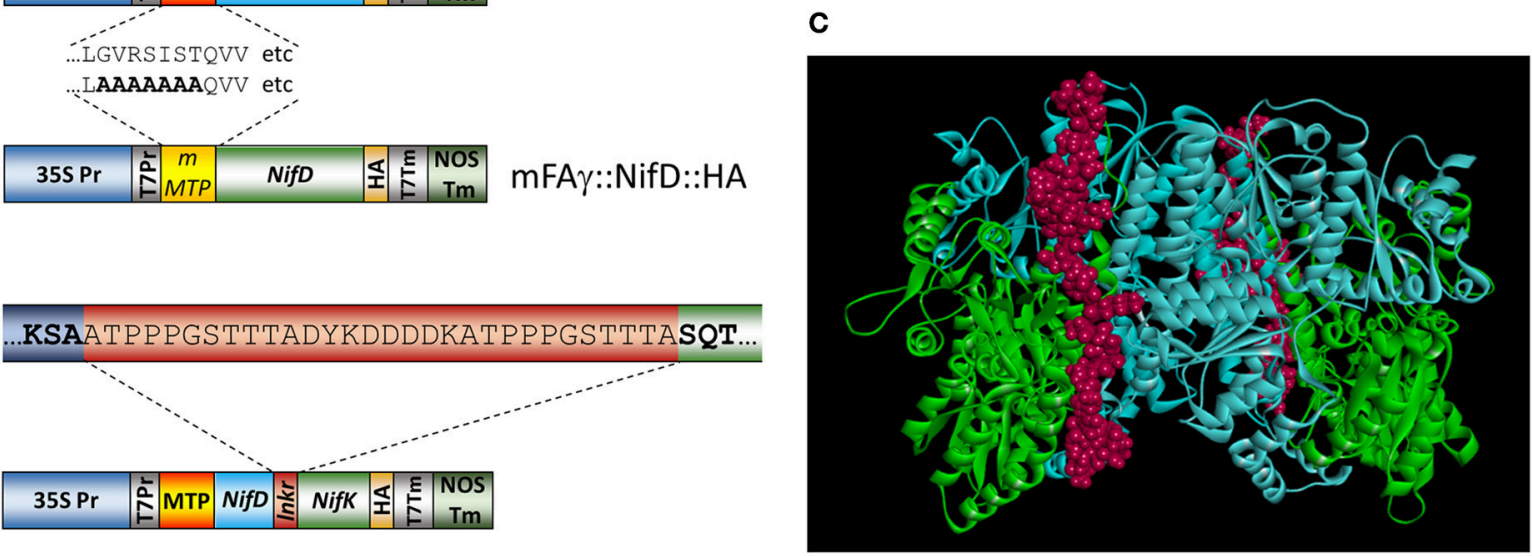

pFA $\gamma::$ NifD-linker- $K_{A t}:: H A$

B

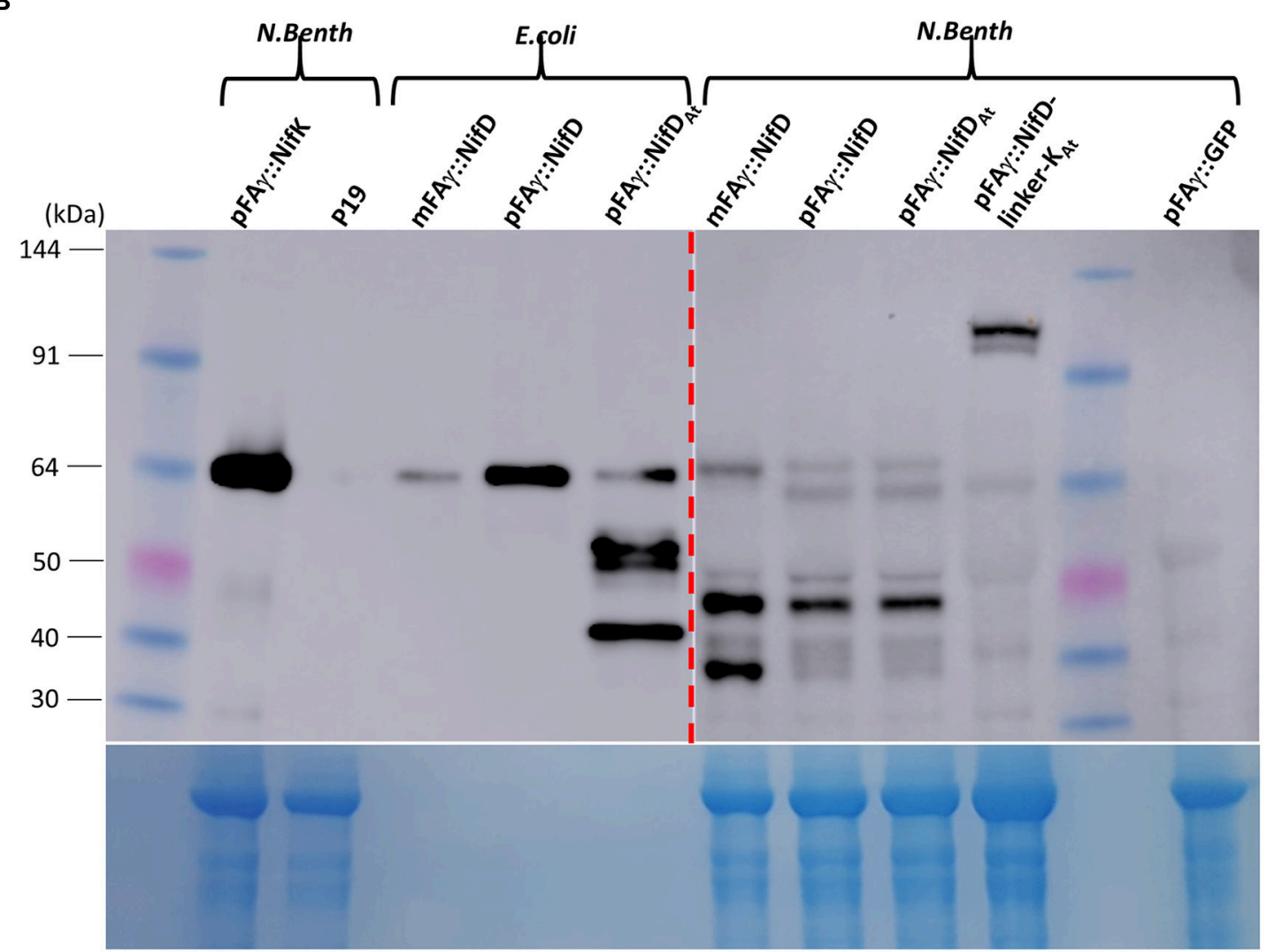

FIGURE 5 | Improved expression of NifD polypeptide fusions in $\mathbf{N}$. benthamiana. (A) Schematics of the various NifD constructs used for expression analysis. Examples of RNA or protein sequence differences are shown; protein sequences for pFA $\gamma:$ NifD::HA and pFA $\gamma:: N_{i f D}$ At::HA are identical, but the codon usages for 
FIGURE 5 | Continued

the NifD coding regions vary, only the first 18 nucleotides with differences in bold red are shown for space. mMTP indicates the region encompassing the mFA $\gamma$ MTP, which contains alanine substitutions identical to mFA $\gamma:$ GFP for disruption of mitochondrial matrix translocation. For $\mathrm{pFA} \gamma:$ NifD-linker-NifK::HA, the entire linker is shown in the red bar, flanked by the NifD (blue) and NifK (green) sequences. (B) Western blot analysis of protein extracts from cells expressing Nif polypeptide fusions and probed with anti-HA. Proteins extracts were prepared from either $E$. coli or $N$. benthamiana indicated by bracketed areas above the blot. The image output levels were adjusted on the right hand side of the blot (shown by red dashed arrow) to prevent oversaturation by $E$. coli bands (original image shown in Supplementary Figure 2). The corresponding Coomassie-stained gel is shown underneath as an indication of protein loading, noting that bacterial extracts have a different total protein profile from that in leaf extracts. (C) In silico representation of the structure of NifD-linker-NifK shown as the $\alpha_{2} \beta_{2}$ heteroteramer. The blue residues are NifD, green residues NifK and the linker displayed as red.

than pFA $\gamma$ (mFA $\gamma::$ NifD::HA) as described earlier (Figure 1A). This construct was made in order to test whether mitochondrial targeting and/or processing, if they occurred, were at least partially responsible for the lack of NifD fusion polypeptide production.

$N$. benthamiana leaves were infiltrated with A. tumefaciens containing these constructs and protein extracts prepared from the infiltrated tissues and analyzed. Encouragingly, the Western blot showed HA-containing bands of the molecular weights expected for both matrix-processed and unprocessed NifD fusion polypeptides when either $\mathrm{pFA} \gamma:: \mathrm{NifD}:: \mathrm{HA}$ or $\mathrm{pFA} \gamma:: \mathrm{NifD}_{\mathrm{At}}:: \mathrm{HA}$ constructs were introduced (Figure 5B). Introduction of mFA $\gamma:: N i f D:: H A$ yielded only the larger (unprocessed) fusion polypeptide, showing that as for mFA $\gamma:: G F P$ (Figure 1A), this modification of the pFA $\gamma$ MTP disrupts matrix processing. Matrix processing was further verified by comparing the position of the bands with that produced from mFA $\gamma:$ NifD::HA and the bacterially produced polypeptides (Figure 5B). The observation of two bands, corresponding in size to processed and unprocessed forms of pFA $\gamma:: \mathrm{NifD}:: \mathrm{HA}$ or $\mathrm{pFA} \gamma:: \mathrm{NifD}_{\mathrm{At}}:: \mathrm{HA}$ indicated that processing of this MTP-NifD fusion polypeptide was not as efficient as for the other MTP-Nif fusion polypeptides as described above. The observed level of the pFA $\gamma:$ NifD::HA or $\mathrm{pFA} \gamma:: \mathrm{NifD}_{\mathrm{At}}:: \mathrm{HA}$ polypeptides were still much lower than the level of the pFA $\gamma::$ NifK::HA fusion polypeptide used as a positive control, despite the same expression construct design and expression conditions. Furthermore, for each of the three modified NifD::HA constructs, additional bands of lower molecular weight were observed on the Western blots, some of which were specific to a particular pFA $\gamma:: N i f D:: H A$ version. For example, an intense band at about $\sim 48 \mathrm{kDa}$ was present for all of the modified NifD::HA constructs, but a different, intense band at about $40 \mathrm{kDa}$ appeared unique when mFA $\gamma:$ NifD::HA was introduced. As these bands were not present for either pFA $\gamma::$ NifK::HA or GFP controls, they could represent NifD::HA degradation products or possibly the product of alternative transcription or translation initiation signals.

\section{Design and Expression of a Translational Fusion between NifD and NifK}

In diazatrophic bacteria the abundance of NifD and NifK are almost equal (Poza-Carrion et al., 2014) and both are found in crystal structures of nitrogenase in a 1:1 ratio as a heterodimer (Schmid et al., 2002). However, it was clear from the above analysis that NifK accumulation was significantly higher than NifD. Given that NifD and NifK form the catalytic unit of nitrogenase, functional activity in plants would likely benefit from a higher level of NifD and a stoichiometric 1:1 NifD:NifK ratio. We therefore designed a translational fusion between NifD and NifK to link the expression of these proteins, as similar recombinant fusion strategies have been shown to improve accumulation (Hondred et al., 1999). This approach required the design of an amino acid linker joining the NifD and NifK units whilst still allowing the proper protein folding of the entire fused D-K polypeptide, as follows. A homology model of the FeMoco protein (NifD and K) $\alpha_{2} \beta_{2}$ hetero-tetramer from $K$. pneumoniae was constructed using the crystal structure of the FeMoco protein complex from Azotobacter vinelandii (PDB ID: 1FP4; Schmid et al., 2002) as a template. In the structural model for the K. pneumoniae $\mathrm{D}_{2} \mathrm{~K}_{2}$ hetero-tetramer, the C-terminus of each NifD subunit was approximately $47 \AA$ from the N-terminus of the NifK partner. Therefore, an unstructured and flexible amino acid linker of 30 residues was designed to span this 47 Å length (Figure 5A; see Section Materials and Methods for full details of the in silico design protocol). The predicted structure of the NifD-linker-NifK composite polypeptide, including its 30amino acid linker, was examined, showing that addition of the linker allowed the full fusion polypeptide to fold into a structure that mimicked the native MoFe complex (Figure 5C). Finally a HA epitope tag was appended to the C-terminus of NifK (as previously for singly expressed NifK) to enable discrimination of the fusion protein with HA antibody, here termed pFA $\gamma:$ NifDlinker-NifK::HA (Figure 5A). When this vector was introduced into $N$. benthamiana cells and protein extracts examined by Western blotting, two specific bands were detected in the size range predicted for the polypeptide at $\sim 120 \mathrm{kDa}$. The closeness of the two bands suggested that both unprocessed and processed forms for the MTP presequence were present. The upper band was of greater intensity than the lower band, suggesting that processing occurred only partially. Nevertheless, the level of accumulation of the pFA $\gamma::$ NifD-linker-NifK::HA polypeptide was greater than for $\mathrm{pFA} \gamma: \mathrm{NifD}: \mathrm{HA}$, but much less than for pFA $\gamma::$ NifK::HA.

\section{Stacking Multiple Nitrogenase Proteins in the Mitochondrial Matrix of Plants}

As nitrogenase activity requires the concerted action of many Nif proteins, it is anticipated that functional nitrogenase reconstitution in plants will require many of the Nif proteins utilized by diazotrophic bacteria for biosynthesis and function. We therefore wanted to determine if multiple Nif proteins could be expressed in $N$. benthamiana using the pFA $\gamma$ MTP. 
To test this concept, genetic constructs encoding four Nif fusion polypeptides of different sizes were chosen that would enable the resultant polypeptides to be identified by Western blot analysis, namely the constructs encoding pFA $\gamma:: \mathrm{NifB}:: \mathrm{HA}$, pFA $\gamma::$ NifS::HA, pFA $\gamma:: N i f H:: H A$, and pFA $\gamma:: N i f Y:: H A$. The four A. tumefaciens cultures transformed with these constructs were mixed in equal amounts and the mixture was then infiltrated into $N$. benthamiana leaves. The accumulated polypeptide levels in this four construct combination were compared to infiltrations with single constructs. It was observed that each polypeptide was more abundant when expressed from a single construct than from the four gene combination (Figure 6). Nevertheless, all four Nif fusion polypeptides were readily detected in the protein extracts from the gene combination, and the molecular weight observed for each polypeptide was identical for the individual and combination infiltrations. This showed that combinations of Nif fusion polypeptides could be produced in the plant cells with the desired targeting and processing of each Nif fusion polypeptide to the mitochondria.

\section{DISCUSSION}

The model diazotroph $K$. pneumoniae requires 16 unique proteins for the biosynthesis and catalytic function of nitrogenase. We have established that this set can be individually expressed as MTP:Nif polypeptide fusions in the mitochondrial matrix of plants, a subcellular location potentially accommodating for nitrogenase function. This is the first practical demonstration of the feasibility of such an approach in plants, and represents important progress toward the aim of engineering plants with nitrogenase activity.

In this study we chose to target Nif proteins to the matrix, primarily because endogenous oxygen consuming enzymes may enable nitrogenase function in a similar manner to the respiratrory protection provided by aerobic diazatrophs (Rey and Maier, 1997). A targeting peptide previously demonstrated to be capable of directing GFP to the Arabidopsis matrix was used for this purpose (Lee et al., 2012), and was relatively long to assist detection of the processed protein (Figure 1). From our analysis, we are confident the chosen MTP targeted most, if not all nitrogenase proteins to the matrix. We make this claim based on several lines of evidence. Firstly, the sizes observed for $N$. benthamiana expressed Nif proteins were consistent with the expected size resulting from matrix peptidase processing (Figures 2, 3). This was also reflected by the difference in size observed between bacterial and plant mitochondrially expressed Nif proteins (NifF and NifZ; Figures 2, 3). Additionally when we mutated the MTP, rendering it incapable of being processed by the mitochondrial import machinery, a larger polypeptide was observed for both NifD and GFP fusion polypeptides, consistent with the difference in size between processed and unprocessed polypeptides (Figures 1, 4). Finally and conclusively, mass spectrometry determined that pFA $\gamma:: \mathrm{NifH}:: \mathrm{HA}$ was cleaved between residues $42-43$ of the MTP as expected for specific processing in the matrix (Figure 2).

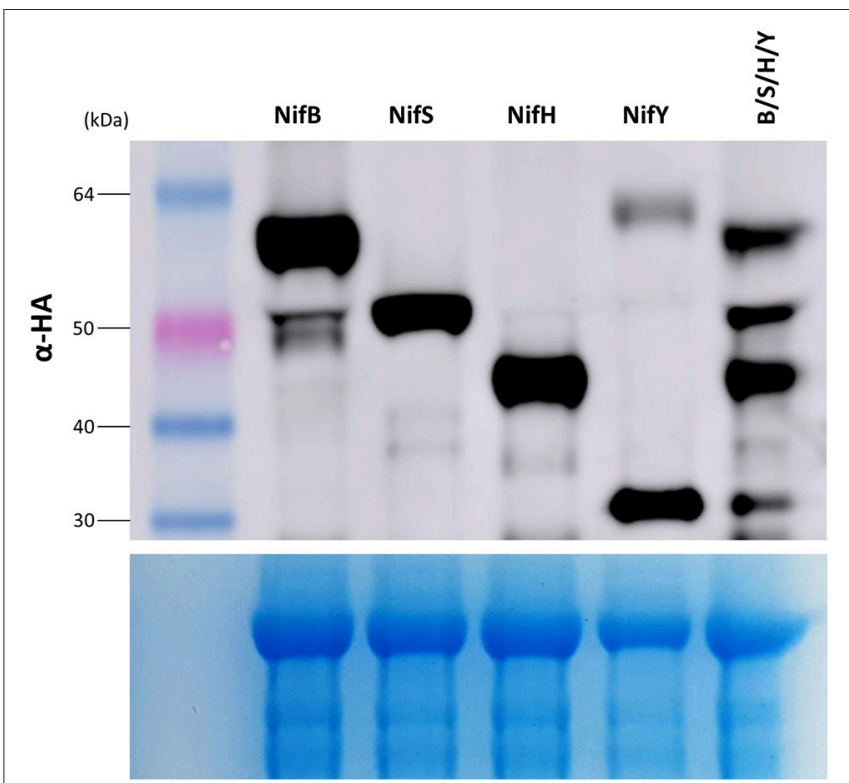

FIGURE 6 | Stacking of multiple Nif proteins in the mitochondrial matrix of $\boldsymbol{N}$. benthamiana. (Upper panel) Western blot of protein extracts for constructs expressing pFA $\gamma:$ Nif::HA either singly (NifB, NifS, NifH, NifY), or as a combination of the same four individual Agrobacterium cultures infiltrated in an equimolar mixture of NifB, S, H, Y. Sizes of markers are indicated on left. The corresponding Coomassie-stained gel is shown in the (lower panel), indicating even loading across all lanes.

The presence of the pFA $\gamma$ MTP did not always lead to complete processing of Nif proteins. In some instances (pFA $\gamma::$ NifX::FLAG, pFA $\gamma::$ Nif::HA, and pFA $\gamma::$ NifD-linker$\mathrm{K}:: \mathrm{HA})$, both processed and unprocessed Nif proteins were observed (Figures 3, 5). Considering there is no consensus sequence for MTPs, and internal protein sequences can influence mitochondrial targeting (Becker et al., 2012), it is perhaps not surprising that we found differences in processing efficiency amongst the Nif proteins. Although, the chosen MTP appears well suited for the majority of Nif protiens, other targeting peptides may need to be explored for efficient processing of NifD, NifD-linker-K, and NifX. Furthermore, additional studies are required to address the consequences of adding MTP-related residues to the $\mathrm{N}$-terminal of Nif proteins with regard to overall function.

Despite use of the strong, constitutive $35 \mathrm{~S}$ promoter, a remarkable degree of variability in protein abundance was observed for the various Nif proteins (Figures 3, 5). Whilst the expression levels of different Nif genes are exquisitely optimized in diazatrophic bacteria such as K. pneumoniae (Temme et al., 2012), it is difficult to predict what the specific requirements will be in N. benthamiana. In this exploratory study, we have focused on finding expression and evidence of processing of each Nif protein, but modifying the expression level of each Nif protein to match bacterial profiles may be required for optimized activity.

Intriguingly, additional higher-molecular-weight protein bands were also found for several of the individual Nif proteins expressed in plants, despite denaturing conditions (see Section 
Materials and Methods). This was most apparent for Nif B, $\mathrm{S}, \mathrm{H}$, and $\mathrm{Y}$; these fainter bands were approximately double the dominant band size, suggestive of a homo-dimerization (Figure 3). In bacteria NifB, S, H, and U function as homodimers in (Yuvaniyama et al., 2000; Rubio and Ludden, 2008). Possibly these additional bands may be higher order structures representative of bacterial functional equivalents. Alternatively, endogenous proteins may interact strongly with transgenic Nif proteins. Shorter peptide fragments of some Nif proteins were also detected via the epitope at the C-terminal of the protein. These fragments may arise due to errant transcription, translation, or protease decay of the mature Nif protein to a shorter size.

Of all the Nif proteins, the essential component required for nitrogenase catalytic activity, NifD, was the most difficult to express as a pFA $\gamma$ fusion (Figures 3, 4). The low levels of pFA $\gamma::$ NifD protein were in contrast to the high levels of $p F A \gamma:: N i f D$ RNA, suggesting translation rates or protein stability were limiting pFA $\gamma::$ NifD protein abundance. Given the critical importance of NifD in catalysis, its requirement to be highly expressed in bacteria (Poza-Carrion et al., 2014), and in an equimolar ratio with NifK, we designed a translational fusion of these two key proteins and found that NifD abundance could be enhanced through this strategy (Figure 5). Given that in bacteria a functional NifD protein requires the interaction of several Nifs (Hu and Ribbe, 2013) the enhancement of NifD we have found by co-translation with $\mathrm{NifK}$ is encouraging for future studies, where further Nif precursor proteins will need to be co-expressed.

This NifD-linker-K fusion also possessed the advantage of being expressed as a single protein, allowing translation of both subunits at the ideal 1:1 ratio, therefore mimicking the stoichiometry of the native heterotetramer. Furthermore, the linker itself was designed to allow sufficient flexibility for the two subunits to form the correct $\alpha_{2} \beta_{2}$ heterotetrameric structure required for catalysis. An earlier report demonstrated that a NifD-K translational fusion without such a flexible linker can impart a limited degree of function (Suh et al., 2003; Lahiri et al., 2005). Therefore, we anticipate our version of NifD-linker-K will at least functionally substitute for individual NifD and NifK expression, possibly with greater efficacy than previously demonstrated. This hypothesis could be tested via a complementation test for nitrogenase activity in bacterial mutants for NifD and NifK.

Re-engineering nitrogenase into plants will ultimately require many Nif proteins to be co-expressed. Here we show that NifB, $\mathrm{NifS}$, NifH, and NifY are at least capable of co-expression within the same tissue (Figure 6). Whilst experiments by others have demonstrated that different proteins with identical MTPs can be targeted to the same mitochondria (Yang et al., 2010), additional experiments will be required to confirm if all these Nifs accumulate together within the same mitochondria. Furthermore, increasing the number of nif genes for transient assays was found to dilute their individual expression, therefore future strategies will need to explore methods to overcome this issue. In this regard, protein fusions such as used here for NifDlinker-K, and multigene cassettes that have been used successfully in previous gene stacking experiments (Naim et al., 2012; Petrie et al., 2014), may be helpful.

Encouragingly, there is emerging evidence that a smaller subset of Nif proteins may be required to achieve functional nitrogenase reconstitution in plant mitochondria. In diazotrophs, genetic approaches have shown that a functional $\mathrm{Fe}$ subunit requires 4 proteins, NifS, NifU, NifM, and NifH. Remarkably only NifH and NifM were required to enable ex vivo Fe protein function from yeast mitochondria (López-Torrejón et al., 2016). Similarly, ex vivo Fe protein activity was achieved by expression of NifH and NifM via transformation of the chloroplast genome (Ivleva et al., 2016). These two reports indicate that in eukaryotic subcellular compartments a biochemically active $\mathrm{Fe}$ subunit can be assembled without the requirement for NifS or NifU transgenes.

These same studies also provide the first experimental evidence that subcellular organelles may be sufficient to support nitrogenase activity, as the Fe subunit is highly sensitive to oxygen. In plant chloroplasts ex vivo Fe protein activity required the lowering of ambient oxygen conditions (Ivleva et al., 2016). By contrast, aerobically grown yeast expressing matrix targeted $\mathrm{NifH}$ and NifM were capable of producing a functional Fe protein (López-Torrejón et al., 2016). Taken together, these two studies indicate subcellular plant organelles may support nitrogenase activity, and the matrix, as an oxygen consuming environment may be the most preferable location.

Although, the MoFe protein is less oxygen sensitive than the Fe subunit (Eady et al., 1972), its biogenesis is undoubtedly more complex. Here we demonstrate that a complete repertoire of Nif proteins can be expressed in plants, including those required for $\mathrm{MoFe}$ subunit assembly, in a subcellular location that is potentially supportive of nitrogenase function. This study adds to the experimental evidence that transgenic plants can be generated to be self-sufficient for bioavailable nitrogen in the future.

\section{MATERIALS AND METHODS}

\section{Construction of Vectors}

Vector pCW441 was designed as dual purpose bacterial and plant expression construct. A region of the pET14b vector (Novogene) was used to design a region of DNA encompassing the T7 promoter, a simple multiple cloning site (Not I and AscI) and the T7 terminator, and this region was chemically synthesized (Geneart) and ligated into a T-DNA-based plant expression vector, pORE1 (Coutu et al., 2007) in between the $35 \mathrm{~S}$ promoter and the NOS terminator regions, generating pCW441. This vector is stable in E. coli, and can produce proteins via the standard T7 polymerase promoter system (Studier and Moffatt, 1986). The $240 \mathrm{bp}$ sequence incorporating the 77 amino acid MTP of the F1-ATPase $\gamma$ subunit (Lee et al., 2012) was chemically synthesized with a NotI and AscI flanking region permitting ligation into pCW441, generating pRA1. Klebsiella oxytcoa Nif genes were codon optimized for eukaryotic expression and commercially synthesized (Geneart). Nif gene sequences were flanked by AscI sites for subcloning and contained either HA or FLAG tags as C terminal fusions 
and designed to allow a translational fusion with the $\mathrm{N}$ terminal MTP. GFP sequence was derived from S65T GFP sequence (Ormö et al., 1996). The full compendium of constructs used in this study are found in Supplementary Table 1.

\section{Growth of Agrobacterium and $N$. benthamiana Infiltrations}

Plant growth and Agrobacterium infiltrations were carried out as described by Naim et al. (2012).

\section{Protein Extractions and Western Blot Analysis}

Infiltrated $N$. benthamina leaf proteins were extracted by grinding an $\sim 2 \times 2 \mathrm{~cm}$ leaf disc in liquid nitrogen then transferring the powder to $300 \mu \mathrm{L}$ of buffer comprised of $125 \mathrm{mM}$ TrisHCL pH 6.8, 4\% SDS, 20\% glycerol, 60 mM DTT. Samples were heated at $95^{\circ} \mathrm{C}$ for $3 \mathrm{~min}$ before centrifugation at $12,000 \mathrm{~g}$ for $2 \mathrm{~min}$. Protein samples $(20 \mu \mathrm{L})$ were separated by SDSPAGE (NuPAGE Bis Tris 4-12\%, Thermofisher.com) at $200 \mathrm{~V}$ for $1 \mathrm{~h}$. Proteins were transferred to PVDF membranes using an iblot system (Thermofisher). After blotting gels were Coomassie stained for $1 \mathrm{~h}$ then rinsed in water for visualization of remaining protein. Membranes were blocked overnight in TBST $+5 \%$ skim milk powder at $4{ }^{\circ} \mathrm{C}$. Anti-HA, and anti-FLAG were purchased from Sigma, anti-GFP was a gift from Leila Blackman (ANU). Antibodies were added at 1:5,000 in TBST with 5\% skim milk powder and incubated for $2 \mathrm{~h}$. Membranes were washed for $3 \times 20$ min with TBST and the secondary antibody [Immun-Star Goat Anti-Mouse (GAM)-HRP conjugate] (Biorad) was added at 1:5,000 in TBST $+5 \%$ skim milk for $1 \mathrm{~h}$, followed by $3 \times 15 \mathrm{~min}$ TBST washes. For secondary antibody detection Amersham ECL reagent was used and membranes were developed either with an $\mathrm{X}$-ray developer or on an Amersham imager (Amerhsam).

\section{Protoplast Preparation, Mitochondria Staining, and Laser-Scanning Confocal Microscopy}

Protoplasts from infiltrated and untransformed N. benthamiana leaves were prepared as described before (Rolland et al., 2016). Mitochondria were stained for 10-20 min using a $100 \mathrm{nM}$ solution of MitoTracker Red CMXRos (ThermoFisher Scientific). Protoplasts were then imaged using an upright Leica SP8 laserscanning confocal microscope with a 40x water immersion objective $(\mathrm{NA}=[1.1])$. GFP was excited at $488 \mathrm{~nm}$ and emission was recorded at 495-520 nm. In the same track, MitoTracker Red CMXRos was excited at $580 \mathrm{~nm}$ and emission was recorded at $595-620 \mathrm{~nm}$. In a separate track, chloroplasts were excited at 633 $\mathrm{nm}$ and emission was recorded at 650-690 $\mathrm{nm}$.

\section{RNA Analysis}

RNA was extracted from infiltrated $N$. benthamina leaves by grinding an $\sim 2 \times 2 \mathrm{~cm}$ leaf disc in liquid nitrogen then transferring the powder to $500 \mu \mathrm{L}$ of Trizol (Life Technologies) buffer for RNA purification. RNA expression analysis was as described previously (Allen et al., 2010) and N. bentahamiana
GADPH was used to normalize gene expression. Primers are described in Supplementary Table 2.

\section{Design of a NifD-Linker-NifK Translational Fusion}

A homology model of the NifD-K $\alpha_{2} \beta_{2}$ heterotetramer from $K$. pneumoniae was constructed using the crystal structure of the NifD-K complex from A. vinelandii (PDB ID: 1FP4) as a template. The C-terminus of $\mathrm{NifD}$ was $\sim 47 \AA$ from the $\mathrm{N}$ terminus of its NifK partner so a linker of appropriate length was designed to connect the two units. The linker was 30 residues in length consisting of an 11-residue section from a known unstructured linker region from Hypocrea jecorina cellobiohydrolase II (Accession no. AAG39980.1) with the final arginine replaced by an alanine, followed by an 8-residue FLAGtag and finally by another copy of the 11-residue unstructured linker sequence with the arginine replaced by an alanine. A geometry optimization and equilibration at constant pressure was carried out on the tetramer using an octahedral TIP3P water box with minimum boundary distance from the solute of 10.0 $\AA$ (without the inclusion of any of the metal centers). This calculation was carried out using Amber 12 (Case et al., 2012) employing the ff99SB force field at $298 \mathrm{~K}$ over $5 \mathrm{~ns}$ in total using 2 fs time steps and SHAKE constraints. The final NifD-linker-NifK sequence is found in Supplementary Table 1.

\section{Tandem Mass Spectrometer Analysis}

Infiltrated $N$. benthamiana tissue was ground in liquid $\mathrm{N}_{2}$ and processed using a Retsch tissuelyser in $2 \mathrm{~mL}$ Eppendorf tubes in $50 \mathrm{mM}$ Tris-HCL pH7.5, $1 \mathrm{mM}$ EDTA, $150 \mathrm{mM}$ $\mathrm{NaCl}, 0.2 \%(\mathrm{w} / \mathrm{v})$ SDS, $10 \%(\mathrm{v} / \mathrm{v})$ glycerol, $5 \mathrm{mM}$ DTT, 0.5 $\mathrm{mM}$ PMSF, and $1 \%(\mathrm{v} / \mathrm{v})$ protease inhibitor cocktail for plants (Sigma). Protein extracts were cleared by centrifugation and used as input for overnight incubation with monoclonal antiHA conjugated agarose beads (Sigma). Unbound proteins were removed by a series of washes with $150 \mathrm{mM}$ Tris-HCL pH 7.5, $5 \mathrm{mM}$ EDTA, $150 \mathrm{mM} \mathrm{NaCl}, 0.1 \%$ (w/v) Triton X-100, 5\% (v/v) glycerol, $5 \mathrm{mM}$ DTT, $0.5 \mathrm{mM}$ PMSF, and $1 \%$ protease inhibitor cocktail for plants (Sigma). Bound proteins were eluted by incubating beads in Laemmli buffer at $95^{\circ} \mathrm{C}$ for $10 \mathrm{~min}$. Input and IP protein samples were separated by SDS-PAGE and the area of gel determined to contain MTP-NifH-HA was determined by Western analysis upon a duplicated sample. From the replicate gel, the corresponding region was excised for ingel tryptic digestion and tandem mass spectrometer analysis as previously described using an Agilent Chip Cube system coupled to an Agilent Q-TOF 6550 mass spectrometer (Campbell et al., 2014). Mass spectra derived from tryptic peptides from common contaminants such as the added trypsin and human keratin were identified before the remaining mass spectral data were used to search against a database containing all protein sequences from Nicotiana species from the NCBInr database (10/3/2015) plus the HA sequence using Spectrum Mill software (Agilent Rev B.04.01.141 SP1) with a precursor mass tolerance of 15 ppm, product mass tolerance of $50 \mathrm{ppm}$, default Q-TOF scoring, and stringent default "autovalidation" settings. Modification of cysteine residues by acrylamide was a required modification and 
oxidation of methionine was allowed as a variable modification. Initially, tryptic cleavage was required and up to two missed cleavages were allowed. After validating peptide matches, the search was repeated with the remaining unmatched spectra allowing non-tryptic cleavage.

\section{AUTHOR CONTRIBUTIONS}

RA Conceived designed and performed experiments, wrote the paper. CW Conceived designed and performed experiments, wrote the paper. VR Designed and performed experiments, helped with writing the paper. PC Performed experiments, helped with writing the paper. KT Designed and performed experiments, helped with writing the paper. AW Designed and performed experiments, helped with writing the paper. SS Helped with experimental design and writing the paper.

\section{REFERENCES}

Allen, R. S., Li, J., Alonso-Peral, M. M., White, R. G., Gubler, F., and Millar, A. A. (2010). MicroR159 regulation of most conserved targets in Arabidopsis has negligible phenotypic effects. Silence 1, 18-18. doi: 10.1186/1758-907X-1-18

Balk, J., and Pilon, M. (2011). Ancient and essential: the assembly of iron-sulfur clusters in plants. Trends Plant Sci. 16, 218-226. doi: $10.1016 /$ j.tplants.2010.12.006

Becker, T., Böttinger, L., and Pfanner, N. (2012). Mitochondrial protein import: from transport pathways to an integrated network. Trends Biochem. Sci. 37, 85-91. doi: 10.1016/j.tibs.2011.11.004

Campbell, P. M., Trueman, H. E., Zhang, Q., Kojima, K., Kameda, T., and Sutherland, T. D. (2014). Cross-linking in the silks of bees, ants and hornets. Insect Biochem. Mol. Biol. 48, 40-50. doi: 10.1016/j.ibmb.2014.02.009

Case, D. A., Darden, T. A., Cheatham, T. E., Simmerling, C. L., Wang, J., Duke, R. E., et al. (2012). AMBER 12. San Francisco, CA: University of California.

Coutu, C., Brandle, J., Brown, D., Brown, K., Miki, B., Simmonds, J., et al. (2007). pORE: a modular binary vector series suited for both monocot and dicot plant transformation. Transgenic Res. 16, 771-781. doi: 10.1007/s11248-007-9066-2

Cui, S., Shi, Y., Groffman, P. M., Schlesinger, W. H., and Zhu, Y.-G. (2013). Centennial-scale analysis of the creation and fate of reactive nitrogen in China (1910-2010). Proc. Natl. Acad. Sci. U.S.A. 110, 2052-2057. doi: $10.1073 /$ pnas. 1221638110

Curatti, L., and Rubio, L. M. (2014). Challenges to develop nitrogenfixing cereals by direct nif-gene transfer. Plant Sci. 225, 130-137. doi: $10.1016 /$ j.plantsci.2014.06.003

De'ath, G., Fabricius, K. E., Sweatman, H., and Puotinen, M. (2012). The 27-year decline of coral cover on the Great Barrier Reef and its causes. Proc. Natl. Acad. Sci. U.S.A. 109, 17995-17999. doi: 10.1073/pnas.1208909109

de Bruijn, F. J. (ed.). (2015). "The quest for biological nitrogen fixation in cereals: a perspective and prospective," in Biological Nitrogen Fixation (Hoboken, NJ: John Wiley \& Sons, Inc.), 1087-1101.

Eady, R. R., Smith, B. E., Cook, K. A., and Postgate, J. R. (1972). Nitrogenase of Klebsiella pneumoniae. Purification and properties of the component proteins. Biochem. J. 128, 655-675. doi: 10.1042/bj1280655

Geddes, B. A., Ryu, M. H., Mus, F., Costas, A. G., Peters, J. W., Voigt, C. A., et al. (2015). Use of plant colonizing bacteria as chassis for transfer of N-2-fixation to cereals. Curr. Opin. Biotechnol. 32, 216-222. doi: 10.1016/j.copbio.2015. 01.004

Glibert, P. M., Maranger, R., Sobota, D. J., and Bouwman, L. (2014). The Haber Bosch-harmful algal bloom (HB-HAB) link. Environ. Res. Lett. 9:105001. doi: 10.1088/1748-9326/9/10/105001

Good, A. G., and Beatty, P. H. (2011). Fertilizing nature: a tragedy of excess in the commons. PLoS Biol. 9:e1001124. doi: 10.1371/journal.pbio.10 01124

\section{ACKNOWLEDGMENTS}

We thank Amratha Menon, Anu Matthew, and Satiya Wati for valuable technical assistance. We thank Greg Tanner for expert advice on protein techniques. We thank Thomas Vanhercke, Iain Wilson, Shoko Okada, and Rob Defeyter for critical reading of the manuscript. We thank Allen Good, Perrin Beatty, and Julia Wong for helpful discussions. RA was supported by a CSIRO OCE Fellowship and ARC DECRA fellowship DE140101886. KT was supported by a CSIRO OCE fellowship.

\section{SUPPLEMENTARY MATERIAL}

The Supplementary Material for this article can be found online at: http://journal.frontiersin.org/article/10.3389/fpls.2017. 00287/full\#supplementary-material

Graf, M., Schoedl, T., and Wagner, R. (2009). "Rationales of gene design and de novo gene construction," in Systems Biology and Synthetic Biology, eds P. Fu and S. Panke (Hoboken, NJ: John Wiley \& Sons, Inc.), 411-438.

Hondred, D., Walker, J. M., Mathews, D. E., and Vierstra, R. D. (1999). Use of ubiquitin fusions to augment protein expression in transgenic plants. Plant Physiol. 119, 713-724. doi: 10.1104/pp.119.2.713

Hu, Y. L., and Ribbe, M. W. (2013). Nitrogenase assembly. Biochim. Biophys. Acta 1827, 1112-1122. doi: 10.1016/j.bbabio.2012.12.001

Huang, S., Taylor, N. L., Whelan, J., and Millar, A. H. (2009). Refining the definition of plant mitochondrial presequences through analysis of sorting signals, Nterminal modifications, and cleavage motifs. Plant Physiol. 150, 1272-1285. doi: 10.1104/pp.109.137885

Ivleva, N. B., Groat, J., Staub, J. M., and Stephens, M. (2016). Expression of active subunit of nitrogenase via integration into plant organelle genome. PLoS ONE 11:e0160951. doi: 10.1371/journal.pone.0160951

Kronzucker, H. J., and Coskun, D. (2015). "Bioengineering nitrogen acquisition in rice: promises for global food security," in Biological Nitrogen Fixation, ed F. J. de Bruijn (Hoboken, NJ: John Wiley \& Sons, Inc.), 47-56.

Lahiri, S., Pulakat, L., and Gavini, N. (2005). Functional NifD-K fusion protein in Azotobacter vinelandii is a homodimeric complex equivalent to the native heterotetrameric MoFe protein. Biochem. Biophys. Res. Commun. 337, 677-684. doi: 10.1016/j.bbrc.2005.09.105

Lee, S., Lee, D. W., Yoo, Y. J., Duncan, O., Oh, Y. J., Lee, Y. J., et al. (2012). Mitochondrial targeting of the Arabidopsis F1-ATPase $\gamma$-subunit via multiple compensatory and synergistic presequence motifs. Plant Cell 24, 5037-5057. doi: $10.1105 /$ tpc.112.105361

Lill, R., and Mühlenhoff, U. (2008). Maturation of iron-sulfur proteins in eukaryotes: mechanisms, connected processes, and diseases. Annu. Rev. Biochem. 77, 669-700. doi: 10.1146/annurev.biochem.76.052705.162653

López-Torrejón, G., Jiménez-Vicente, E., Buesa, J. M., Hernandez, J. A., Verma, H. K., and Rubio, L. M. (2016). Expression of a functional oxygen-labile nitrogenase component in the mitochondrial matrix of aerobically grown yeast. Nat. Commun. 7:11426. doi: 10.1038/ncomms11426

Merrick, M., and Dixon, R. (1984). Why dont plants fix nitrogen. Trends Biotechnol. 2, 162-166. doi: 10.1016/0167-7799(84)90034-9

Mueller, N. D., Gerber, J. S., Johnston, M., Ray, D. K., Ramankutty, N., and Foley, J. A. (2012). Closing yield gaps through nutrient and water management. Nature 490, 254-257. doi: 10.1038/nature11420

Murcha, M. W., Wang, Y., Narsai, R., and Whelan, J. (2014). The plant mitochondrial protein import apparatus - The differences make it interesting. Biochem. Biophys. Acta 1840, 1233-1245. doi: 10.1016/j.bbagen.2013.09.026

Naim, F., Nakasugi, K., Crowhurst, R. N., Hilario, E., Zwart, A. B., Hellens, R. P., et al. (2012). Advanced engineering of lipid metabolism in Nicotiana benthamiana using a draft genome and the V2 viral silencing-suppressor protein. PLoS ONE 7:e52717. doi: 10.1371/journal.pone.0052717 
Oldroyd, G. E., and Dixon, R. (2014). Biotechnological solutions to the nitrogen problem. Curr. Opin. Biotechnol. 26, 19-24. doi: 10.1016/j.copbio.2013.08.006

Ormö, M., Cubitt, A. B., Kallio, K., Gross, L. A., Tsien, R. Y., and Remington, S. J. (1996). Crystal structure of the Aequorea victoria green fluorescent protein. Science 273, 1392-1395. doi: 10.1126/science.273.5280.1392

Petrie, J. R., Shrestha, P., Belide, S., Kennedy, Y., Lester, G., Liu, Q., et al. (2014). Metabolic engineering Camelina sativa with fish oil-like levels of DHA. PLoS ONE 9:e85061. doi: 10.1371/journal.pone.0085061

Poza-Carrion, C., Jimenez-Vicente, E., Navarro-Rodriguez, M., Echavarri-Erasun, C., and Rubio, L. M. (2014). Kinetics of nif gene expression in a nitrogen-fixing bacterium. J. Bacteriol. 196, 595-603. doi: 10.1128/JB.00942-13

Rey, L., and Maier, R. J. (1997). Cytochrome c terminal oxidase pathways of Azotobacter vinelandii: analysis of cytochrome c4 and c5 mutants and upregulation of cytochrome c-dependent pathways with N2 fixation. J. Bacteriol. 179, 7191-7196. doi: 10.1128/jb.179.22.7191-7196.1997

Robson, R. L., and Postgate, J. R. (1980). Oxygen and hydrogen in biological nitrogen-fixation. Annu. Rev. Microbiol. 34, 183-207. doi: 10.1146/annurev.mi.34.100180.001151

Rockstrom, J., Steffen, W., Noone, K., Persson, A., Chapin, F. S., Lambin, E. F., et al. (2009). A safe operating space for humanity. Nature 461, 472-475. doi: $10.1038 / 461472 \mathrm{a}$

Rolland, V., Badger, M. R., and Price, G. D. (2016). Redirecting the cyanobacterial bicarbonate transporters BicA and SbtA to the chloroplast envelope: soluble and membrane cargos need different chloroplast targeting signals in plants. Front. Plant Sci. 7:185. doi: 10.3389/fpls.2016.00185

Rubio, L. M., and Ludden, P. W. (2008). Biosynthesis of the ironmolybdenum cofactor of nitrogenase. Annu. Rev. Microbiol. 62, 93-111. doi: 10.1146/annurev.micro.62.081307.162737

Santi, C., Bogusz, D., and Franche, C. (2013). Biological nitrogen fixation in non-legume plants. Ann. Bot. 111, 743-767. doi: 10.1093/aob/mct048

Schmid, B., Ribbe, M. W., Einsle, O., Yoshida, M., Thomas, L. M., Dean, D. R., et al. (2002). Structure of a cofactor-deficient nitrogenase MoFe protein. Science 296, 352-356. doi: 10.1126/science. 1070010

Seefeldt, L. C., Hoffman, B. M., and Dean, D. R. (2009). Mechanism of Mo-dependent nitrogenase. Annu. Rev. Biochem. 78, 701-722. doi: 10.1146/annurev.biochem.78.070907.103812

Smil, V. (2002). Nitrogen and food production: proteins for human diets. Ambio 31, 126-131. doi: 10.1579/0044-7447-31.2.126
Studier, F. W., and Moffatt, B. A. (1986). Use of bacteriophage T7 RNA polymerase to direct selective high-level expression of cloned genes. J. Mol. Biol. 189, 113-130. doi: 10.1016/0022-2836(86)90385-2

Suh, M.-H., Pulakat, L., and Gavini, N. (2003). Functional expression of a fusiondimeric mofe protein of nitrogenase in Azotobacter vinelandii. J. Biol. Chem. 278, 5353-5360. doi: 10.1074/jbc.M208969200

Sutton, M. A., Erisman, J. W., Dentener, F., and Moller, D. (2008). Ammonia in the environment: from ancient times to the present. Environ. Pollut. 156, 583-604. doi: 10.1016/j.envpol.2008.03.013

Temme, K., Zhao, D., and Voigt, C. A. (2012). Refactoring the nitrogen fixation gene cluster from Klebsiella oxytoca. Proc. Natl. Acad. Sci. U.S.A. 109, 7085-7090. doi: 10.1073/pnas.1120788109

Wood, C. C., Petrie, J. R., Shrestha, P., Mansour, M. P., Nichols, P. D., Green, A. G., et al. (2009). A leaf-based assay using interchangeable design principles to rapidly assemble multistep recombinant pathways. Plant Biotechnol. J. 7, 914-924. doi: 10.1111/j.1467-7652.2009.00453.x

Yang, J., Liu, X., Yang, X., and Zhang, M. (2010). Mitochondriallytargeted expression of a cytoplasmic male sterility-associated orf220 gene causes male sterility in Brassica juncea. BMC Plant Biol. 10:231. doi: 10.1186/1471-2229-10-231

Yuvaniyama, P., Agar, J. N., Cash, V. L., Johnson, M. K., and Dean, D. R. (2000). NifS-directed assembly of a transient [2Fe-2S] cluster within the NifU protein. Proc. Natl. Acad. Sci. U.S.A. 97, 599-604. doi: 10.1073/pnas.97.2.599

Conflict of Interest Statement: This work is subject to a patent by CSIRO in which RA, KT, AW and CW are inventors.

The other authors declare that the research was conducted in the absence of any commercial or financial relationships that could be construed as a potential conflict of interest.

Copyright (C) 2017 Allen, Tilbrook, Warden, Campbell, Rolland, Singh and Wood. This is an open-access article distributed under the terms of the Creative Commons Attribution License (CC BY). The use, distribution or reproduction in other forums is permitted, provided the original author(s) or licensor are credited and that the original publication in this journal is cited, in accordance with accepted academic practice. No use, distribution or reproduction is permitted which does not comply with these terms. 